\title{
Electrokinetic flow in an elliptic microchannel covered by ion-penetrable membrane
}

\author{
Jyh-Ping Hsu ${ }^{\mathrm{a}, *}$, Ying-Lun Weng ${ }^{\mathrm{a}, 1}$, Duu-Jong Lee ${ }^{\mathrm{a}, 1}$, Shiojenn Tseng ${ }^{\mathrm{b}}$, \\ Ay Su ${ }^{\mathrm{c}}$, Chur-Jen Chen ${ }^{\mathrm{d}}$ \\ a Department of Chemical Engineering, National Taiwan University, Taipei 10617, Taiwan \\ ${ }^{\mathrm{b}}$ Department of Mathematics, Tamkang University, Tamsui, Taipei 25137, Taiwan \\ ${ }^{c}$ Department of Mechanical Engineering \& Fuel Cells Research Center, Yuan Ze University, 135 Yuan-Tung Road, \\ Chung Li, Tao Yuan 320, Taiwan \\ ${ }^{\mathrm{d}}$ Department of Mathematics, Tunghai University, Taichung 403, Taiwan \\ Received 6 June 2006; received in revised form 22 July 2006; accepted 4 August 2006 \\ Available online 22 August 2006
}

\begin{abstract}
The electrokinetic flow of an electrolyte solution in an elliptical microchannel covered by an ion-penetrable, charged membrane layer is examined theoretically. The present analysis extends previous results in that a two-dimensional problem is considered, and the system under consideration simulates the flow of a fluid, for example, in a microchannel of biological nature such as vein. The electroosmostic volumetric flow rate, the total electric current, the streaming potential, and the electroviscous effect of the system under consideration are evaluated. We show that, for a constant hydraulic diameter, the variations of these quantities as a function of the aspect ratio of a microchannel may have a local minimum or a local maximum at a medium level of ionic strength, which depends on the thickness of the membrane layer. For a constant cross-sectional area, the electroosmostic volumetric flow rate, the total electric current, and the streaming potential increase monotonically with the increase in the aspect ratio, but the reverse is true for the electroviscous effect.
\end{abstract}

(C) 2006 Elsevier B.V. All rights reserved.

Keywords: Electrokinetic flow; Elliptic microchannel; Ion-penetrable membrane; Streaming current; Electroosmotic velocity

\section{Introduction}

The transport phenomena occur in a microchannel have various applications in modern technologies involving a micro-sized system, and have drawn the attention of researches in various fields. Microchannel emulsification, for instance has been justified to be promising for producing monodispersed emulsions [1-5]. New experimental techniques are also continuously proposed for the production of monodispersed emulsions based on microchannels [6-10]. Microchannel has also been adopted as a liquid-liquid extraction device in analytical chemistry [11-14]. Microreactor, which have many desirable properties, is another typical application of a microchannel device [15-19].

\footnotetext{
* Corresponding author. Fax: +886223623040.

E-mail address:.jphsu@ntu.edu.tw (J.-P. Hsu).

1 Fax: +886223623040.
}

Microchannels have also been used in fuel cell relevant technologies [20-25].

In addition to a pressure gradient, an electric field is often applied to drive the fluid flow through a microchannel. Here, the charged conditions on the microchannel wall and the concentration of electrolytes in the liquid phase play the key roles. Although the electrokinetic flow in a microchannel has been investigated extensively [e.g., 26-31] relevant studies remain active. In general, except under drastic assumptions, such as low surface potential, constant charged properties, and simple geometries, the governing equations and the associated boundary conditions need to be solved numerically.

Most of the previous studies concerning the flow in a microchannel focused on the case where the wall of a microchannel is of rigid nature. Although this is appropriate in many industrial applications, it can be unrealistic in some other cases such as surfaces of biological nature and inorganic surfaces with an adsorbed polymer layer. In practice, these types of surface are 
simulated by a rigid surface covered by an ion-penetrable membrane layer, which contains dissociable functional groups [32]. In this case, instead of spreading over a surface, the fixed charge is distributed in a finite volume in space, and solving the governing equations becomes more complicated since both the electric and the flow fields inside and outside a membrane layer need be considered. Several attempts have been made to model the electrokinetic flow in a microchannel having a non-rigid surface, including, for instance, cylindrical [33], planar slit [34-36], and rectangle microchannels [37].

In this study the flow of an electrolyte solution in an elliptic microchannel, the surface of which is covered by an ionpenetrable membrane, is analyzed theoretically. Numerical simulations are conducted to examine the effect of the key parameters on the flow behavior under consideration. In particular, the influences of the ionic strength, the thickness of membrane layer, and the geometry of a channel are discussed.

\section{Theory}

Referring to Fig. 1(a), we consider a steady flow of an $a: b$ electrolyte solution, $a$ and $b$ being, respectively, the valences of cations and anions, in an elliptic microchannel of length $L$, major axis $2 W$, and minor axis $2 H$. The surface of the microchannel is covered by an ion-penetrable membrane layer of thickness $d$, which carries fixed charge. Suppose that $L$ is sufficiently long so that the end effects can be neglected. The cartesian coordinates $(x, y, z)$ are adopted with its origin located at the center of the microchannel. Because the flow of liquid is in the $z$-direction, only the $z$-component of the fluid velocity $V, u(x, y)$, needs be considered. An electric field $E_{z}$ with magnitude $E_{z}=-(\partial \psi / \partial z)$ in the $z$-direction and a pressure gradient $P$ with magnitude $P=-(\partial p / \partial z)$ in the same direction are applied, where $\psi$ and $p$ are, respectively, the electrical potential and the pressure. The liquid phase has constant dielectric constant $\varepsilon$ and viscosity $\eta$. As illustrated in Fig. 1(b), the symmetric nature of the present problem suggests that only the quarter of the cross-section of the microchannel, $\Omega \in\left[x^{2} / W^{2}+y^{2} / H^{2} \leq 1 \cap x \geq 0 \cap y \geq 0\right]$, needs be considered. $\Omega_{1}, \Omega_{2}$, and $\Omega_{3}$ are the boundaries of $\Omega$, and $\Omega_{4}$ represents the membrane-liquid phase interface.

\subsection{Electric field}

The electric potential $\psi(x, y, z)$ comprises that arising from the applied electric field $-E_{z} z$ and that arising from the charged membrane $\phi(x, y)$. We have

$\psi(x, y, z)=\phi(x, y)-E_{z} z$

For the present case, $\phi(x, y)$ can be described by

$\frac{\partial^{2} \phi}{\partial x^{2}}+\frac{\partial^{2} \phi}{\partial y^{2}}=\frac{-\left(\rho_{\mathrm{el}}+j w F C_{0}\right)}{\varepsilon}, \quad(x, y) \in \Omega$

where $C_{0}$ is density of the fixed charge in the membrane layer and $w$ is its valence, $\rho_{\mathrm{el}}$ the space charge density of mobile ions, and $j$ is a region index $(j=0$ for liquid phase, $j=1$ for membrane layer). We assume that both $C_{\mathrm{a}}$ and $C_{\mathrm{b}}$ follow a Boltzmann
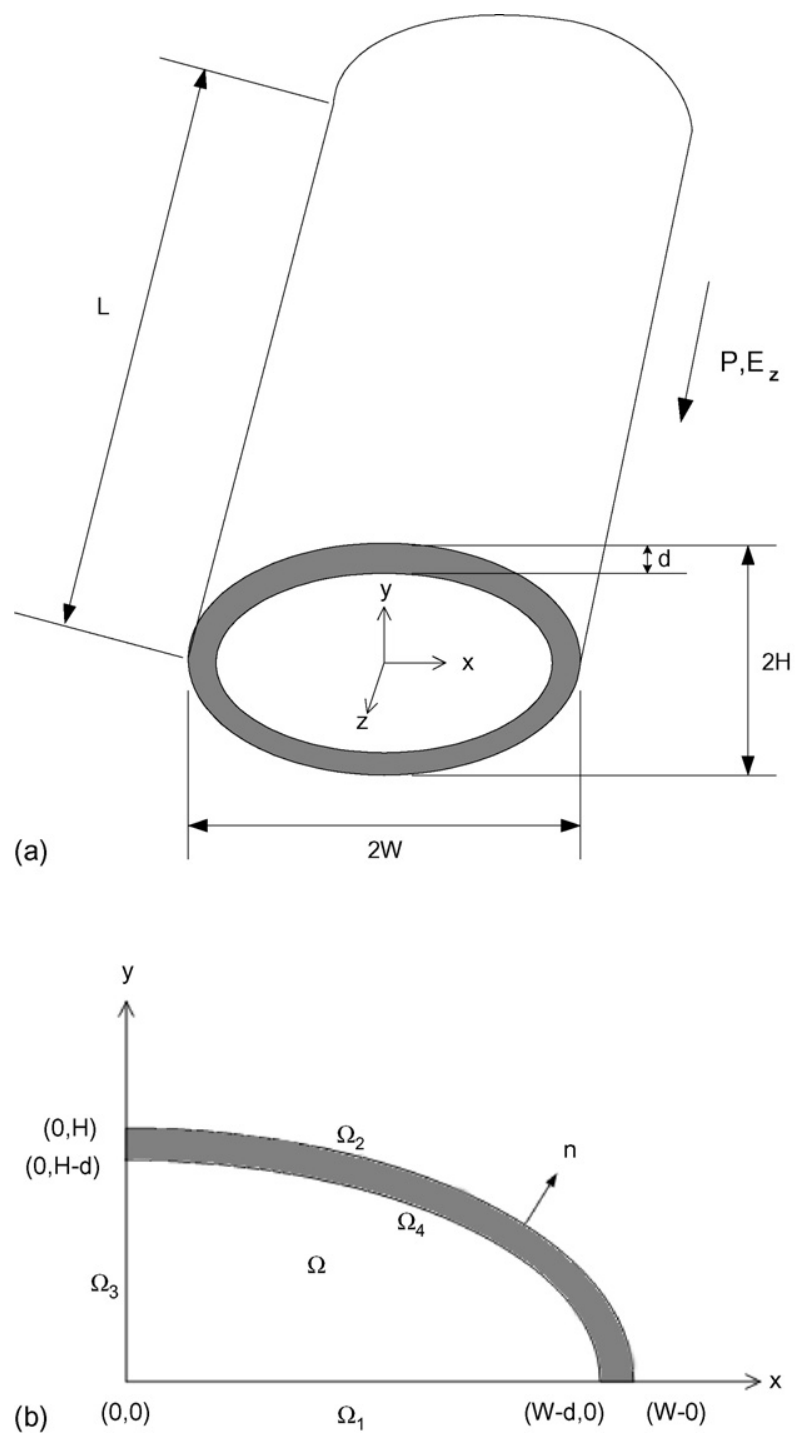

Fig. 1. Schematic representation of the system under consideration (a) and computational domain (b)

distribution, that is,

$C_{\mathrm{a}}=C_{\mathrm{a}}^{0} \exp \left(\frac{-a F \phi}{R T}\right)$

$C_{\mathrm{b}}=C_{\mathrm{b}}^{0} \exp \left(\frac{b F \phi}{R T}\right)$

where $C_{\mathrm{a}}^{0}$ and $C_{\mathrm{b}}^{0}$ are, respectively, the bulk concentrations of cations and anions, $F$ and $R$ are, respectively, the Faraday constant and the gas constant, and $T$ is the absolute temperature. Since electroneutrality must be satisfied, we have $a C_{\mathrm{a}}^{0}=b C_{\mathrm{b}}^{0}$. The space charge density for mobile ions $\rho_{\mathrm{el}}$ can be expressed as

$\rho_{\mathrm{el}}=-a C_{\mathrm{a}}^{0} F\left[\exp \left(\frac{b F \phi}{R T}\right)-\exp \left(\frac{-a F \phi}{R T}\right)\right]$ 
For a more concise representation, Eq. (2) is rewritten is scaled symbols as

$\frac{\partial^{2} \Phi}{\partial X^{2}}+\frac{\partial^{2} \Phi}{\partial Y^{2}}=K^{2} \frac{G+j N}{a+b}, \quad(X, Y) \in \Omega$

where $\quad \Phi=F \phi / R T, \quad X=x / D_{\mathrm{h}}, \quad Y=y / D_{\mathrm{h}}, \quad D=d / D_{\mathrm{h}}, \quad K$ $=\kappa D_{\mathrm{h}}, \quad G=\exp (b \Phi)-\exp (-a \Phi), \quad N=-w C_{0} / a C_{\mathrm{a}}^{0}=$ $-(a+b) w C_{0} / 2 I, \quad I=a(a+b) C_{\mathrm{a}}^{0} / 2, \quad$ and $\quad D_{\mathrm{h}}=\pi H / \int_{0}^{\pi / 2}$ $\sqrt{1-\left[1-(H / W)^{2}\right] \sin ^{2} \theta} \mathrm{d} \theta$. Here, $I$ and $D_{\mathrm{h}}$ are, respectively, the ionic strength and the hydraulic diameter of a microchannel, and the Debye-Huckel parameter $\kappa$ is defined by

$\kappa=\left(\frac{a C_{\mathrm{a}}^{0}(a+b) F^{2}}{\varepsilon R T}\right)^{1 / 2}=\left(\frac{2 I F^{2}}{\varepsilon R T}\right)^{1 / 2}$

The boundary conditions associated with Eq. (2) are assumed as

$\nabla \phi \cdot \boldsymbol{n}=0, \quad(x, y) \in \Omega_{1}, \Omega_{3}$

$\phi_{\Omega_{4}^{+}}=\phi_{\Omega_{4}^{-}}$

$\nabla \phi \cdot \boldsymbol{n}_{\Omega_{4}^{+}}=\nabla \phi \cdot \boldsymbol{n}_{\Omega_{4}^{-}}$

$\nabla \phi \cdot \boldsymbol{n}=0, \quad(x, y) \in \Omega_{2}$

where $\nabla$ is the gradient operator and $\boldsymbol{n}$ is the unit normal vector. Eq. (7a) arises from the symmetric nature of the present problem. Eqs. (7b) and (7c) imply that both the electric potential and the electric field are continuous across the membrane-liquid interface. Note that if the dielectric constant of the membrane layer $\varepsilon_{1}$ is different from that of the bulk liquid phase $\varepsilon_{2}$, then Eq. (2) should be modified accordingly, and a correction factor $\varepsilon^{\prime}=\varepsilon_{1} / \varepsilon_{2}$ needs be added to the right-hand side of Eq. (7c). For simplicity, we assume $\varepsilon^{\prime}=1$. Eq. (7d) suggests that the electric field vanishes on the rigid core of a microchannel. In terms of scaled symbols, Eqs. (7a)-(7d) become

$$
\begin{aligned}
& \nabla \Phi \cdot \boldsymbol{n}=0, \quad(X, Y) \in \Omega_{1}, \Omega_{3} \\
& \Phi_{\Omega_{4}^{+}}=\Phi_{\Omega_{4}^{-}} \\
& \nabla \Phi \cdot \boldsymbol{n}_{\Omega_{4}^{+}}=\nabla \Phi \cdot \boldsymbol{n}_{\Omega_{4}^{-}} \\
& \nabla \Phi \cdot \boldsymbol{n}=0, \quad(X, Y) \in \Omega_{2}
\end{aligned}
$$

\subsection{Flow field}

For incompressible fluid, $u(x, y)$ can be described by the Navier-Stokes equation

$\frac{\partial^{2} u}{\partial x^{2}}+\frac{\partial^{2} u}{\partial y^{2}}=\frac{-\left[-(\partial p / \partial z)+\rho_{\mathrm{el}} E_{z}-j \gamma u\right]}{\eta}, \quad(x, y) \in \Omega$

where $\gamma$ is friction coefficient per unit volume of the membrane layer. This expression can be rewritten in scaled form as

$$
\frac{\partial^{2} U}{\partial X^{2}}+\frac{\partial^{2} U}{\partial Y^{2}}=K^{2}\left[L \frac{G}{a+b}-M+j \lambda^{2} U\right], \quad(X, Y) \in \Omega
$$

where $U=u / u_{0}, L=\varepsilon R T E_{z} / \eta F u_{0}, \lambda^{2}=\gamma / \eta \kappa^{2}$, and $M=P / \eta \kappa^{2} u_{0}$, $u_{0}$ being a reference velocity. The boundary conditions associated with Eq. (9) are assumed as

$\nabla u \cdot \boldsymbol{n}=0, \quad(x, y) \in \Omega_{1}, \Omega_{3}$

$u_{\Omega_{4}^{+}}=u_{\Omega_{4}^{-}}$

$\nabla u \cdot \boldsymbol{n}_{\Omega_{4}^{+}}=\nabla u \cdot \boldsymbol{n}_{\Omega_{4}^{-}}$

$u=0, \quad(x, y) \in \Omega_{2}$

Eq. (11a) arises from the symmetric nature of the system, and Eqs. (11b) and (11c) imply that both the velocity and the shear stress are continuous across the membrane-liquid interface. Note that if the viscosity of the liquid in the membrane phase $\eta_{1}$ is different from that of the bulk liquid phase $\eta_{2}$, Eq. (9) should be modified accordingly, and a correction factor $\eta^{\prime}=\eta_{1} / \eta_{2}$ needs be added to the right-hand side of Eq. (11c). Under typical conditions, however, because the porosity of membrane is large [38], we assume that $\eta_{1} \cong \eta_{2}$, and, therefore, $\eta^{\prime}=1$. Eq. (11d) arises from the non-slip nature of rigid wall. In terms of scaled symbols, Eqs. (11a)-(11c) become

$\nabla U \cdot \boldsymbol{n}=0, \quad(X, Y) \in \Omega_{1}, \Omega_{3}$

$U_{\Omega_{4}^{+}}=U_{\Omega_{4}^{-}}$

$\nabla U \cdot \boldsymbol{n}_{\Omega_{4}^{+}}=\nabla U \cdot \boldsymbol{n}_{\Omega_{4}^{-}}$

$U=0, \quad(X, Y) \in \Omega_{2}$

\subsection{Volumetric flow rate}

Since we assume that the membrane layer is negatively charged, cations (counterions) move in the $z$-direction and anions (coions) move in the $-z$-direction as a response to the applied electric field. Also, because the concentration of cations in the double layer is higher than that of anions, there is a net flow of liquid in the $z$-direction. The volumetric flow rate arising from the applied electric field can be evaluated by

$$
\begin{aligned}
V_{t} & =4 \iint_{\Omega} u \mathrm{~d} \Omega \\
& =4 \int_{0}^{H} \int_{0}^{\sqrt{\left(1-y^{2} / H^{2}\right) W^{2}}} u(x, y) \mathrm{d} x \mathrm{~d} y, \quad P=0
\end{aligned}
$$

In terms of scaled symbols, we have

$$
\begin{aligned}
V_{t}= & 4 D_{\mathrm{h}}^{2} u_{0} \int_{0}^{H / D_{\mathrm{h}}} \int_{0}^{\sqrt{\left(1-y^{2} / H^{2}\right) W^{2}} / D_{\mathrm{h}}} \\
& \times U(X, Y) \mathrm{d} X \mathrm{~d} Y, \quad M=0
\end{aligned}
$$

\subsection{Total electric current}

In our case the current density $i(x, y)$ can be calculated by

$i(x, y)=a F C_{\mathrm{a}}(x, y) v_{\mathrm{a}}(x, y)-b F C_{\mathrm{b}}(x, y) v_{\mathrm{b}}(x, y)$ 
where $C_{\mathrm{a}}(x, y)$ and $C_{\mathrm{b}}(x, y)$ are, respectively, the concentrations of cations and anions, and $v_{\mathrm{a}}(x, y)$ and $v_{\mathrm{b}}(x, y)$ are, respectively, the velocities of cations and anions. The electrochemical potentials of cations and anions, $\mu_{\mathrm{a}}$ and $\mu_{\mathrm{b}}$, can be expressed respectively as

$\mu_{\mathrm{a}}(x, y, z)=\mu_{\mathrm{a} 0}+\operatorname{ae} \psi(x, y, z)+k_{\mathrm{b}} T \ln \left[C_{\mathrm{a}}(x, y)\right]$

$\mu_{\mathrm{b}}(x, y, z)=\mu_{\mathrm{b} 0}-\operatorname{be} \psi(x, y, z)+k_{\mathrm{b}} T \ln \left[C_{\mathrm{b}}(x, y)\right]$

where $\mu_{\mathrm{a} 0}$ and $\mu_{\mathrm{b} 0}$ are constant. Because $E_{z}$ is in the $z$-direction, $\psi(x, y, z)$ can be expressed as

$\psi(x, y, z)=\phi(x, y)-E_{z} z$
The velocity of ionic species $m, v_{m}(x, y)$, can be expressed as

$v_{m}(x, y)=u(x, y)-\frac{1}{f_{m}} \frac{\partial}{\partial z} \mu_{m}$

where $f_{m}$ is the drag coefficient of ionic species $m, m=a, b$. Substituting Eqs. (16a)-(16c) into Eq. (17) and applying Eq. (15), we obtain

$$
i(x, y)=\rho u(x, y)+\frac{F^{2} E_{z}}{N_{0}}\left[\frac{a^{2}}{f_{\mathrm{a}}} C_{\mathrm{a}}(x, y)+\frac{b^{2}}{f_{\mathrm{b}}} C_{\mathrm{b}}(x, y)\right]
$$

(a)
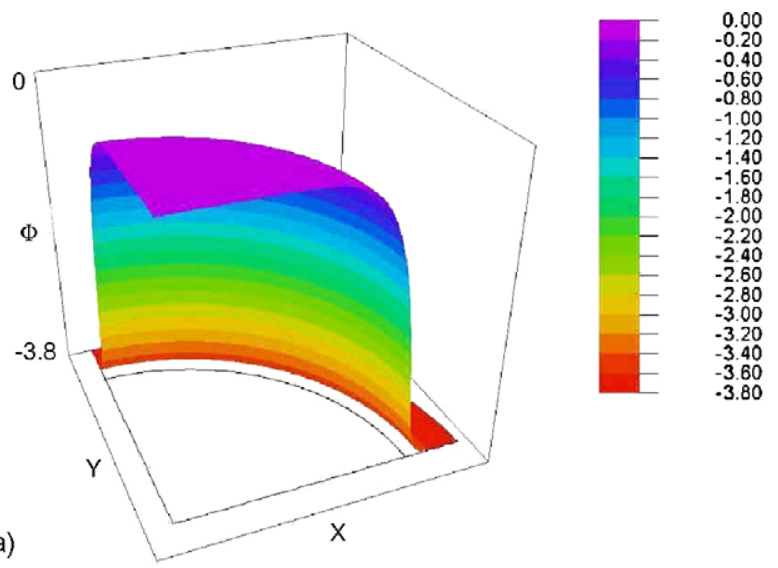

(b)
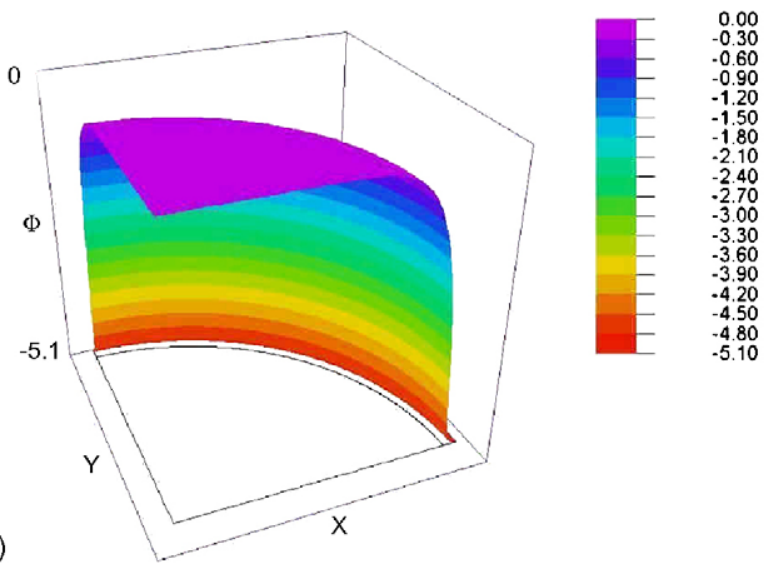

(c)
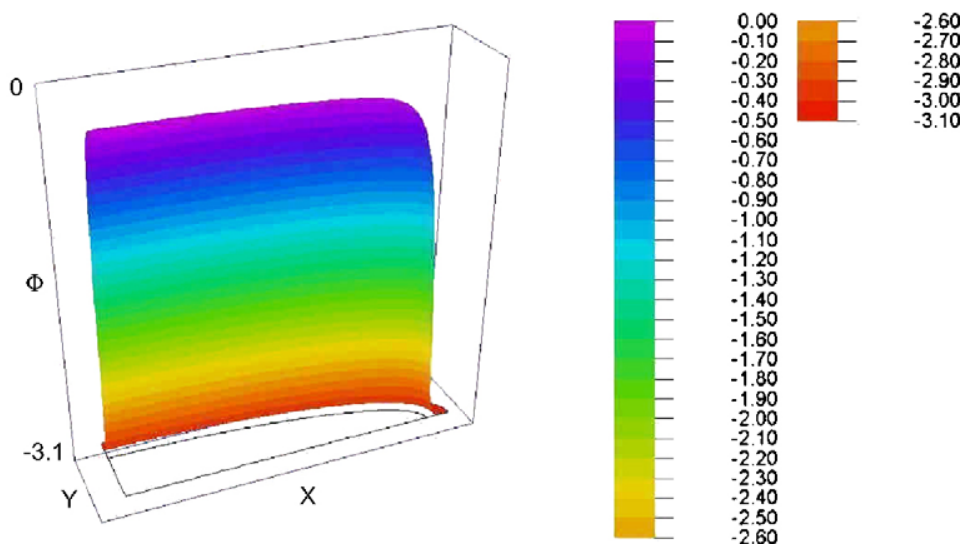

Fig. 2. Simulated spatial variation of scaled potential at $I=10^{-4} \mathrm{M}, P=10^{7} \mathrm{~N} / \mathrm{m}^{3}, D_{\mathrm{h}}=10^{-6} \mathrm{~m}$, and $E_{z}=0 \mathrm{~V} / \mathrm{m}$. (a) $H / W=1$ and $d=D_{\mathrm{h}} / 10$, (b) $H / W=1$ and $d=D_{\mathrm{h}} / 40$, and (c) $H / W=1 / 4$ and $d=D_{\mathrm{h}} / 10$. 
The first term on the right-hand side of this expression represents the convection current and the second term denotes the conduction current. Eq. (18) can be rewritten in scaled form as

$$
\begin{aligned}
i(X, Y)= & \frac{-F s C_{0}}{N}\left\{-G u_{0} U+\frac{F E_{z}}{N_{0}}\left[\frac{a}{f_{\mathrm{a}}} \exp (-a \Phi)\right.\right. \\
& \left.\left.+\frac{b}{f_{\mathrm{b}}} \exp (b \Phi)\right]\right\}
\end{aligned}
$$

where $N_{0}$ is the Avogadro's number. The total current $I_{\mathrm{t}}$, which is in the $z$-direction, can be evaluated by

$$
\begin{aligned}
I_{\mathrm{t}} & =4 \iint_{\Omega} i \mathrm{~d} \Omega=4 \int_{0}^{H} \int_{0}^{\sqrt{\left(1-y^{2} / H^{2}\right) W^{2}}} i(x, y) \mathrm{d} x \mathrm{~d} y \\
& =4 D_{\mathrm{h}}^{2} \int_{0}^{H / D_{\mathrm{h}}} \int_{0}^{\sqrt{\left(1-y^{2} / H^{2}\right) W^{2}} / D_{\mathrm{h}}} i(X, Y) \mathrm{d} X \mathrm{~d} Y
\end{aligned}
$$

\section{Results and discussion}

The performance of the elliptical microchannel considered is examined through numerical simulation. The equations governing the electric field and the flow field and the associated boundary conditions are solved by FlexPDE [39], a commercial software based on finite element method. For illustration, we assume that the liquid phase contains 1:1 electrolyte, and the membrane layer is negatively charged with $w=-1$. The value of $\gamma$ in Eq. (9) is estimated by the modified Rouse theory [40] using polydimethylsiloxane, which predicts that $\gamma=10^{13}$ $\left(\mathrm{N} \mathrm{s} / \mathrm{m}^{4}\right)$. According to Mala et al. [41], if the bulk concentration of electrolyte is in the range $10^{-4}$ to $10^{-6} \mathrm{M}$, then the zeta potential ranges from 100 to $200 \mathrm{mV}$. Therefore, the amount of fixed charge per unit length of membrane layer is assumed the value of $10^{-7} \mathrm{C} / \mathrm{m}$. Also, we assume $f_{\mathrm{a}}=f_{\mathrm{b}}=10^{-12} \mathrm{~kg} / \mathrm{s}$, $T=298 \mathrm{~K}, \varepsilon=7.08 \times 10^{-10} \mathrm{C} /(\mathrm{V} \mathrm{m}), \eta=9 \times 10^{-4} \mathrm{~kg} /(\mathrm{m} \mathrm{s})$, and $u_{0}=10^{-3} \mathrm{~m} / \mathrm{s}$.

Fig. 2 shows the simulated spatial variation of the scaled electrical potential $\Phi$ for various combinations of the aspect ratio $(H / W)$ and the thickness of the membrane layer $d$. The corresponding spatial variations of the scaled velocity $U$ are illustrated in Fig. 3. Fig. 2(a) and (b) indicates that for a fixed value of $(H / W)$, the thinner the membrane layer, the larger the $|\Phi|$, and Fig. 2(a) and (c) reveal that for a fixed membrane thickness, the larger the $(H / W)$, the larger the $|\Phi|$. A comparison between Fig. 3(a) and (c) shows that for a fixed value of $d$, the magnitude of the liquid velocity at the center of a microchannel declines with the decrease in $(H / W)$. This is because if the value of $D_{\mathrm{h}}$ is fixed, the smaller the $(H / W)$ the narrower a microchannel, the more significant the non-slip effect of microchannel wall, and therefore, the slower the velocity of fluid. A comparison between Fig. 3(a) and (b) indicates that for a fixed value of $(H / W)$, the thinner the membrane layer the greater the magnitude of the liquid velocity at the center of a microchannel. This is because the thinner the membrane layer is the smaller the resistance for liquid flow through it. As illustrated in Fig. 4, if the value of $D_{\mathrm{h}}$

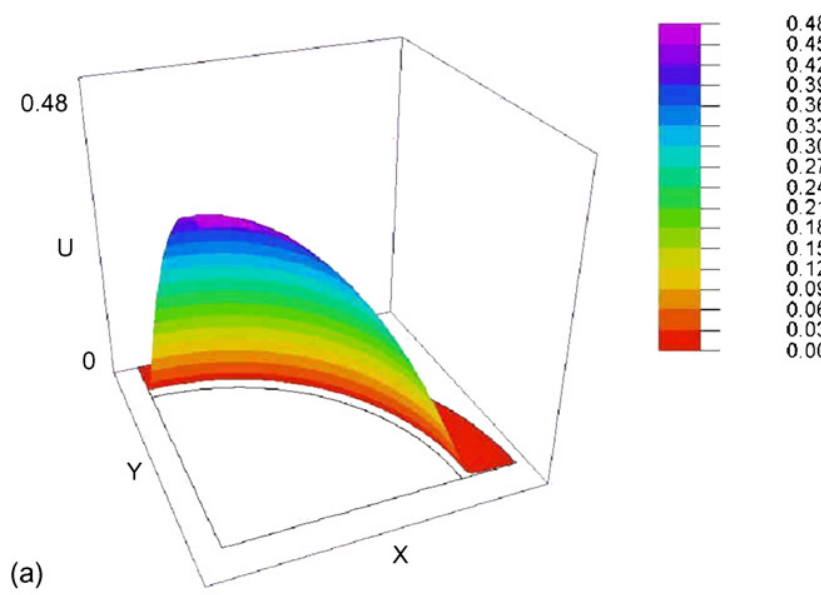

0.48
0.45
0.42
0.39
0.36
0.33
0.30
0.27
0.24
0.21
0.18
0.15
0.12
0.09
0.06
0.03
0.00

(b)



(c)
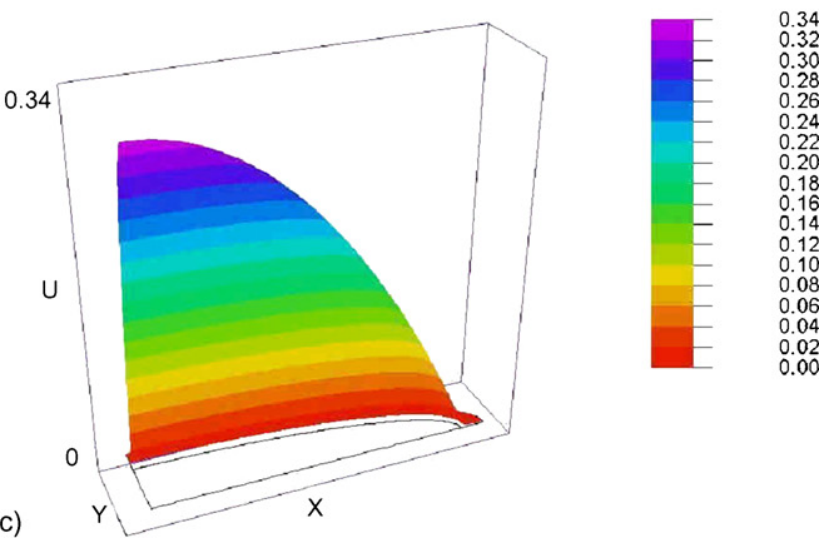

Fig. 3. Simulated spatial variation of scaled velocity $U$ for the case of Fig. 2 .

is fixed, the smaller the $(H / W)$ the narrower a microchannel, but the larger its cross-sectional area.

Fig. 5 shows the variation of the total current $I_{\mathrm{t}}$ as a function of the aspect ratio $(H / W)$ at various combinations of $I$ and $d$ for the case when no pressure gradient is applied. This figure reveals that for fixed values of $(H / W)$ and $d, I_{\mathrm{t}}$ increases with the increase in $I$, and if the value of $I$ is fixed, the thinner the membrane the larger the $I_{\mathrm{t}}$. The former is because the concentration of mobile ions increases with the increase in $I$, and the latter is because the thinner the membrane the smaller the resistance for the movement of ions. Note that if pressure gradient is not applied, both cations 

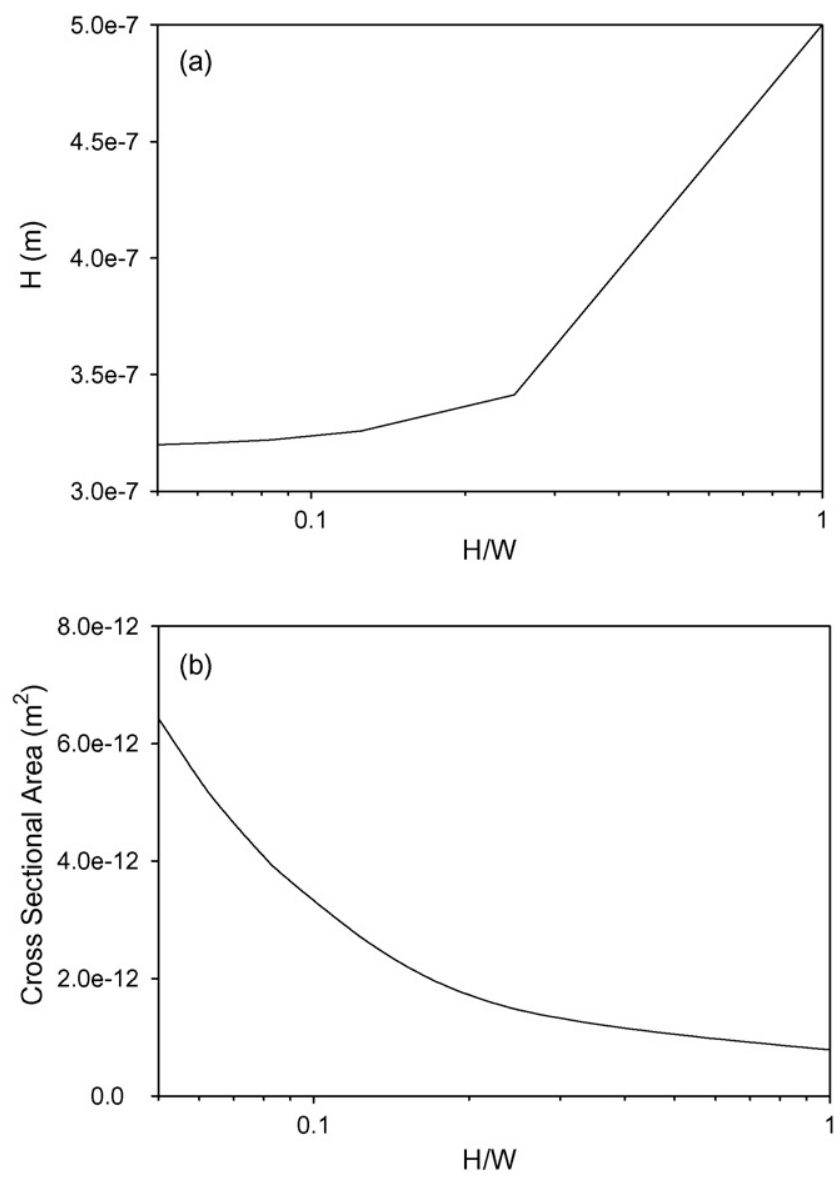

Fig. 4. Variation of semi-minor axis $H$ (a) and cross-sectional area (b) as a function of aspect ratio for the case when $D_{\mathrm{h}}=10^{-6} \mathrm{~m}$.

and anions contribute positively to $I_{\mathrm{t}}$. Therefore, although the higher the level of $I$ the thinner the double layer and the less significant the contribution to $I_{\mathrm{t}}$ by the ions inside the double layer, due to the hydrodynamic resistance of the membrane layer, the contribution of ions in the bulk liquid phase dominates. For a fixed level of $I$ (or thickness of double layer), the thinner the membrane layer the less the amount of double layer it covers, that is, the more the amount of ionic species outside the membrane layer. Since the ions outside a membrane layer move easier than those inside the thinner the membrane layer the larger the $I_{\mathrm{t}}$. Fig. 5(a) suggests that if $I$ is sufficiently large, $I_{\mathrm{t}}$ decreases with the increase in $(H / W)$; but the reverse is true if $I$ is small, as shown in Fig. 5(c). For a medium value of $I, I_{\mathrm{t}}$ may have a local minimum as $(H / W)$ varies, as illustrated in Fig. 5(b). This is because if $D_{\mathrm{h}}$ is fixed, the smaller the $(H / W)$ the narrower a microchannel is, the more significant the no-slip effect of its wall, that is, the more significant the viscous effect, and therefore, the smaller the $I_{\mathrm{t}}$. On the other hand, for a fixed value of $D_{\mathrm{h}}$, the smaller the $(H / W)$ the larger the cross-sectional area of a microchannel, the greater the amount of ions inside, and the larger the $I_{\mathrm{t}}$. Therefore, if $I$ is sufficiently large, the effect of cross-sectional area dominates, and the smaller the $(H / W)$ the larger the $I_{\mathrm{t}}$, and the reverse is true if $I$ is not large enough. Fig. 5(b) indicates that the thicker the membrane layer the larger the $(H / W)$ at which the minimum of $I_{\mathrm{t}}$ occurs. This is because the thicker the membrane
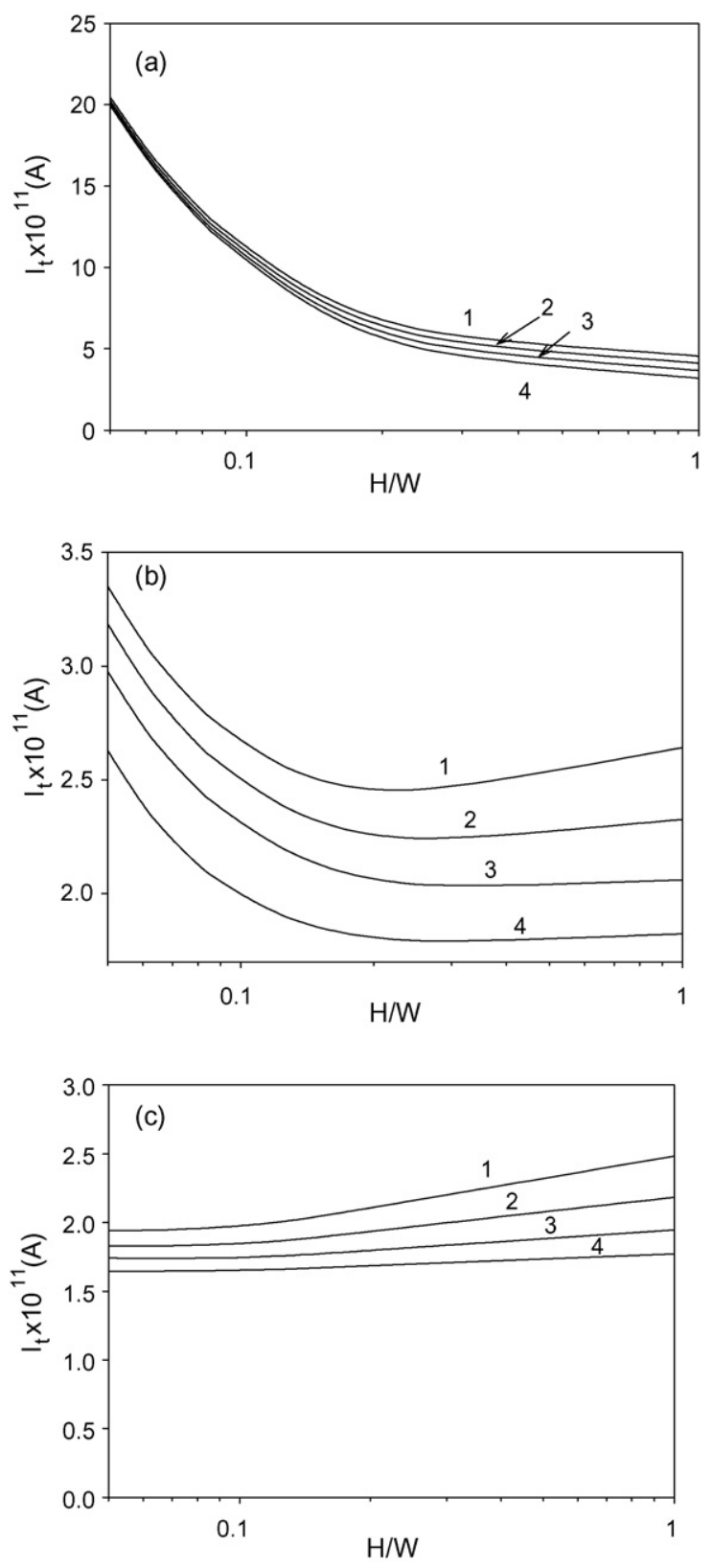

Fig. 5. Variation of total current $I_{\mathrm{t}}$ as a function of aspect ratio $(H / W)$ for various ionic strength $I$ and membrane thickness $d$ at $D_{\mathrm{h}}=10^{-6} \mathrm{~m}, E_{z}=1000 \mathrm{~V} / \mathrm{m}$, and $P=0 \mathrm{~N} / \mathrm{m}^{3}$. (a) $I=10^{-3} \mathrm{M}$, (b) $I=10^{-4} \mathrm{M}$ and (c) $I=10^{-5} \mathrm{M}$. Curve 1 , $d=D_{\mathrm{h}} / 40 ; 2, d=D_{\mathrm{h}} / 20 ; 3, d=D_{\mathrm{h}} / 10 ; 4, d=D_{\mathrm{h}} / 4$.

layer the more significant its hydrodynamic resistance, which is equivalent to raising the viscous effect of the microchannel wall, and therefore, the minimum of $I_{\mathrm{t}}$ occurs earlier.

Fig. 6 shows the variation of the total volumetric flow rate $V_{\mathrm{t}}$ as a function of $(H / W)$ for the case of Fig. 5. Fig. 6 suggests that for fixed values of $(H / W)$ and $d$, the smaller the $I$ the larger the $V_{\mathrm{t}}$, and for a fixed value of $I$, the smaller the $d$ the larger the $V_{\mathrm{t}}$. Since pressure gradient is not applied, the contribution of coions to $V_{\mathrm{t}}$ is reverse to that of counterions. Also, because the latter dominates, we focused on the influence of counterions. The smaller the value of $I$ the thicker the double layer, the more widely the distribution of counterions, and since the greater 

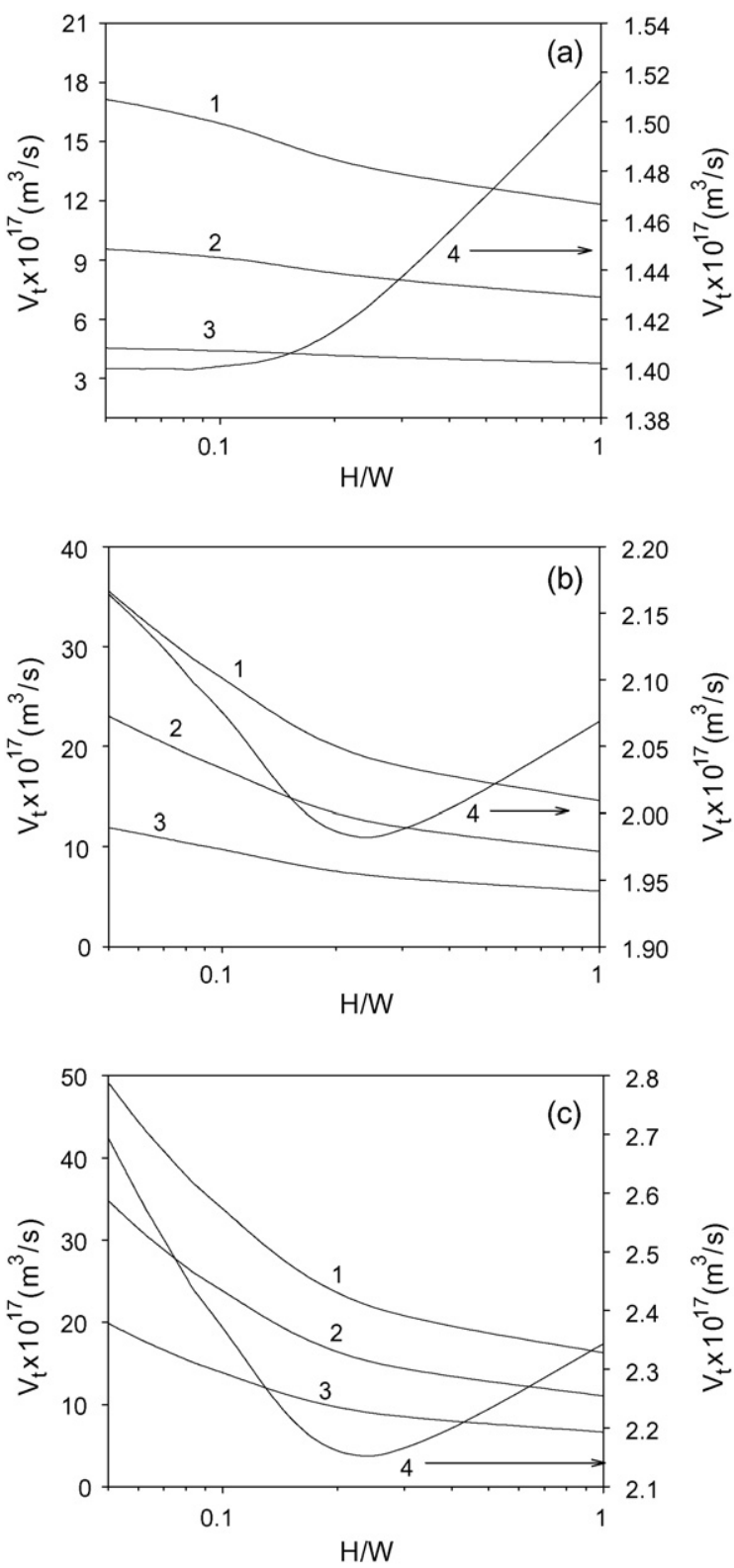

Fig. 6. Variation of total volumetric flow rate current $V_{\mathrm{t}}$ as a function of aspect ratio $(H / W)$ at various $I$ and $d$ for the case of Fig. 5 .

the amount of counterions can be driven by the applied electric field, $V_{\mathrm{t}}$ is lager. For a thinner membrane layer, because the corresponding hydrodynamic residence is smaller, $V_{\mathrm{t}}$ becomes larger. Also, as discussed in Fig. 5, if the value of $I$ is fixed, since the amount of counterions in a thinner membrane layer is greater than that in a thicker one $V_{\mathrm{t}}$ of the former is larger than that of the latter. As can be seen in Fig. 6(a), for the case when $I$ is larger, if the membrane layer is thin, $V_{\mathrm{t}}$ declines with the increase in $(H / W)$, and the reverse is true if the membrane layer is sufficiently thick. If $I$ is large, the counterions in the double layer are close to the wall of a microchannel. Since the viscous effect of the wall is significant, the contribution of those counterions to $V_{\mathrm{t}}$ is unimportant. In this case, if the membrane layer is sufficiently thick, then the increase in the cross-sectional area of a microchannel as $(H / W)$ decreases is less significant than the corresponding increase in the viscous effect due to the presence of the microchannel wall, and therefore, $V_{\mathrm{t}}$ increases with the increase in $(H / W)$. On the other hand, if the membrane layer is thin, the effect of the increase in the cross-sectional area of a microchannel as $(H / W)$ decreases becomes more significant, and $V_{\mathrm{t}}$ declines with the increase in $(H / W)$. Fig. 6(b) and (c) indicate that if $I$ is small and the membrane layer is thin, $V_{\mathrm{t}}$ declines with the increase in $(H / W)$. However, if the membrane layer is sufficiently thick, $V_{\mathrm{t}}$ exhibits a local minimum as $(H / W)$ varies. If $I$ is small, the counterions in the double layer are more widely distributed, which implies that more counterions can be driven by
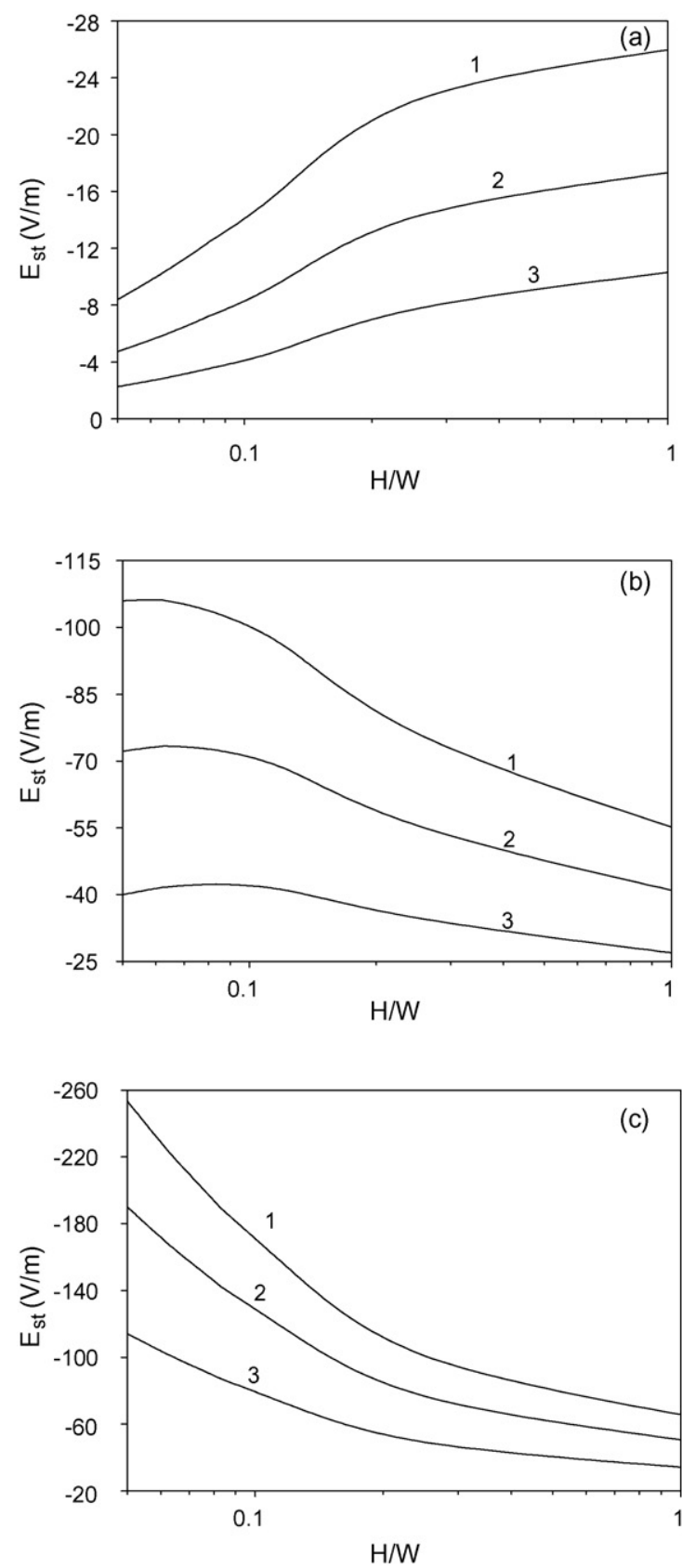

Fig. 7. Variation of streaming potential $E_{\mathrm{st}}$ as a function of aspect ratio $(H / W)$ for various ionic strength $I$ and membrane thickness $d$ at $D_{\mathrm{h}}=10^{-6} \mathrm{~m}$ and $P=10^{7} \mathrm{~N} / \mathrm{m}^{3}$. (a) $I=10^{-3} \mathrm{M}$, (b) $I=10^{-4} \mathrm{M}$ and (c) $I=10^{-5} \mathrm{M}$. Curve 1 , $d=D_{\mathrm{h}} / 40 ; 2, d=D_{\mathrm{h}} / 20 ; 3, d=D_{\mathrm{h}} / 10$. 

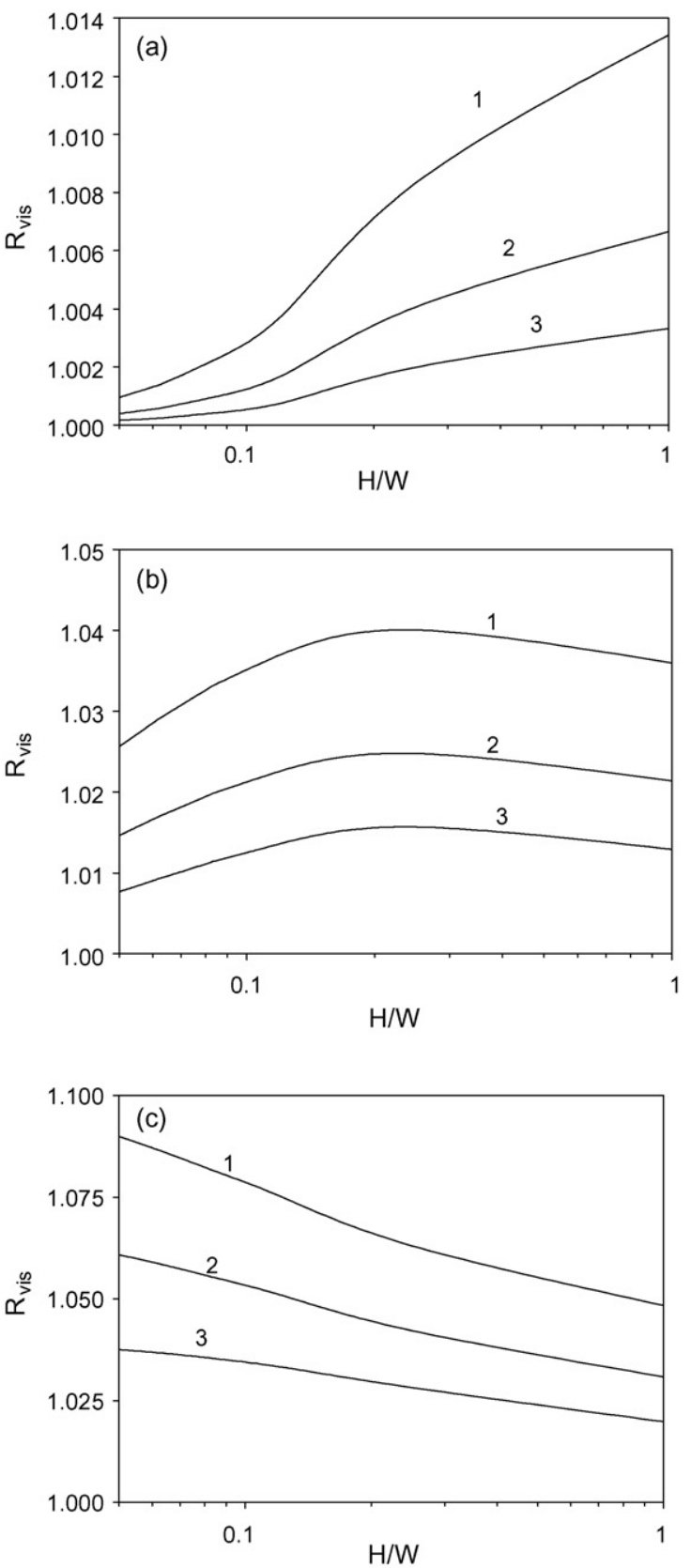

Fig. 8. Variation of streaming potential $R_{\mathrm{vis}}$ as a function of aspect ratio $(H / W)$ at various $I$ and $d$ for the case of Fig. 7.

the applied electric field, and the contribution of double layer to $V_{\mathrm{t}}$ is more important. Also, if counterions are widely distributed, the effect of the cross-sectional area is more significant than the viscous effect, and therefore, $V_{\mathrm{t}}$ declines with the increase in $(H / W)$. As can be seen in Fig. 4 , if the value of $D_{\mathrm{h}}$ is fixed, $H$ is insensitive to the variation of $(H / W)$ when it is small. On the other hand, the cross-sectional area is sensitive to the variation of $(H / W)$ when it is small. These imply that the influence of the viscous effect is more important when $(H / W)$ is large and that of the cross-sectional area is more significant when $(H / W)$ is small. We conclude that if the membrane layer is thin, regardless of the level of ionic strength, the effect the cross-sectional area is significant. On the other hand, if it is thick, the relative significance of the viscous effect and the effect of the cross-sectional area depends upon the level of ionic strength.

For the case when only a pressure gradient is applied, the streaming potential $E_{\mathrm{st}}$ is in the $-z$-direction. Fig. 7 shows the variation of $E_{\mathrm{st}}$ as a function of the aspect ratio $(H / W)$ at various combinations of $I$ and $d$. The corresponding variation of $R_{\mathrm{vis}}$ $\left(=\eta_{\mathrm{a}} / \eta\right)$ a measure for the electroviscous effect, is presented in Fig. 8, $\eta_{\mathrm{a}}$ and $\eta$ being, respectively, the apparent viscosity of the liquid phase when the presence of double layer is considered and that when it is neglected. For the present case although both coions and counterions contribute positively to $V_{\mathrm{t}}$, the contribution of counterions to $I_{\mathrm{t}}$ is in the $z$-direction and that of coions is in the $-z$-direction. Fig. 7 shows that if the value of $(H / W)$ is fixed, both a decrease in $I$ and that in $d$ lead to an increase in $\left|E_{\mathrm{st}}\right|$. The former is because the smaller the value of $I$, the wider the distribution of counterions, a larger amount of counterions can be driven by the applied pressure gradient, which yields a larger $I_{\mathrm{t}}$, and a larger $\left|E_{\mathrm{st}}\right|$ is necessary to balance this total current. For a thinner membrane layer, since the corresponding hydrodynamic retardation is smaller, ions are easier to be driven by the applied pressure gradient, and therefore, both $\left|E_{\mathrm{st}}\right|$ and $R_{\mathrm{vis}}$ become larger. Figs. 7 and 8 reveal that if $I$ is large, both $\left|E_{\mathrm{st}}\right|$ and $R_{\mathrm{vis}}$ increase with the increase in $(H / W)$; if $I$ is small, both $\left|E_{\mathrm{st}}\right|$ and $R_{\text {vis }}$ decline with the increase in $(H / W)$; for an intermediate value of $I$, both $\left|E_{\text {st }}\right|$ and $R_{\text {vis }}$ have a local maximum as $(H / W)$ varies. As in the case of Fig. 6, these behaviors are the net result of the competition between the effects of the thickness of double layer, the cross-sectional area of a microchannel, and the viscous arising from microchannel wall. For the case only a pressure gradient is applied, if $I$ is large, the viscous effect dominates, since $I_{\mathrm{t}}$ increases with the increase in $(H / W), E_{\mathrm{st}}$ increases accordingly. On the other hand, if $I$ is small, the effect of cross-sectional area dominates, $I_{\mathrm{t}}$ declines with the increase in $(H / W)$, so is $\left|E_{\mathrm{st}}\right|$. For a medium level of $I$, if $(H / W)$ is small, the viscous effect dominates and the effect of cross-section area dominates if $(H / W)$ is large. These lead to a local maximum in $\left|E_{\mathrm{st}}\right|$ as $(H / W)$ varies. Since $V_{\mathrm{t}}$ is influenced by the induced electric field, the larger the $\left|E_{\mathrm{st}}\right|$ the smaller the $V_{\mathrm{t}}$, which yields a larger $R_{\mathrm{vis}}$, and therefore, the behavior of $R_{\mathrm{vis}}$ as $(H / W)$ varies is similar to that of $V_{\mathrm{t}}$.

Fig. 9 shows the variation of $R_{\text {vis }}$ as a function of the electrokinetic diameter $D_{\mathrm{e}}\left(=\kappa D_{\mathrm{h}}\right)$ at various values of $(H / W)$. This figure

Table 1

Variation of $\left|\phi_{\mathrm{w}}\right|$ at various combinations of ionic strength $I$ and $D_{\mathrm{e}}\left(=\kappa D_{\mathrm{h}}\right)$ for the case when $D_{\mathrm{h}}=10^{-6} \mathrm{~m}, E_{z}=1000 \mathrm{~V} / \mathrm{m}, P=0 \mathrm{~N} / \mathrm{m}^{3}, d=D_{\mathrm{h}} / 10$, and $H / W=1$

\begin{tabular}{lcccccc}
\hline$I(\mathrm{M})$ & $1.00 \times 10^{-3}$ & $5.50 \times 10^{-4}$ & $1.00 \times 10^{-4}$ & $1.00 \times 10^{-5}$ & $5.00 \times 10^{-6}$ & $2.50 \times 10^{-6}$ \\
$D_{\mathrm{e}}=\kappa D_{\mathrm{h}}$ & 103.004 & 76.390 & 32.571 & 10.301 & 7.284 & 7.150 \\
$\left|\Phi_{\mathrm{w}}\right|$ & 1.37 & 1.92 & 3.6 & 5.9 & 6.6 & 170 \\
$\left|\phi_{\mathrm{w}}\right|(\mathrm{mV})$ & 35.1 & 49.3 & 92.5 & 150 & 190 \\
\hline
\end{tabular}




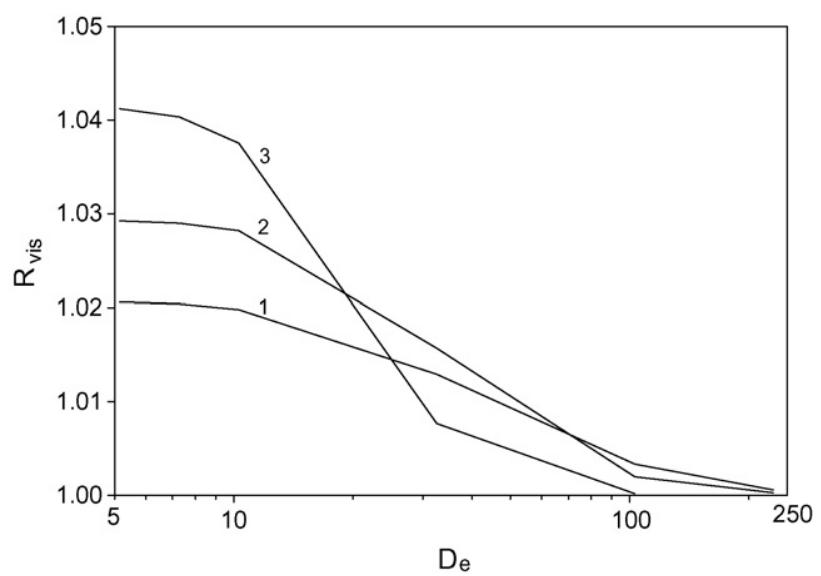

Fig. 9. Variation of electroviscous parameter $R_{\mathrm{vis}}$ as a function of electrokinetic diameter $D_{\mathrm{e}}$ for various aspect ratio $(H / W)$ at $D_{\mathrm{h}}=10^{-6} \mathrm{~m}, d=D_{\mathrm{h}} / 10$, and $P=10^{7} \mathrm{~N} / \mathrm{m}^{3}$. Curve $1, H / W=1 ; 2, H / W=1 / 4 ; 3, H / W=1 / 20$.

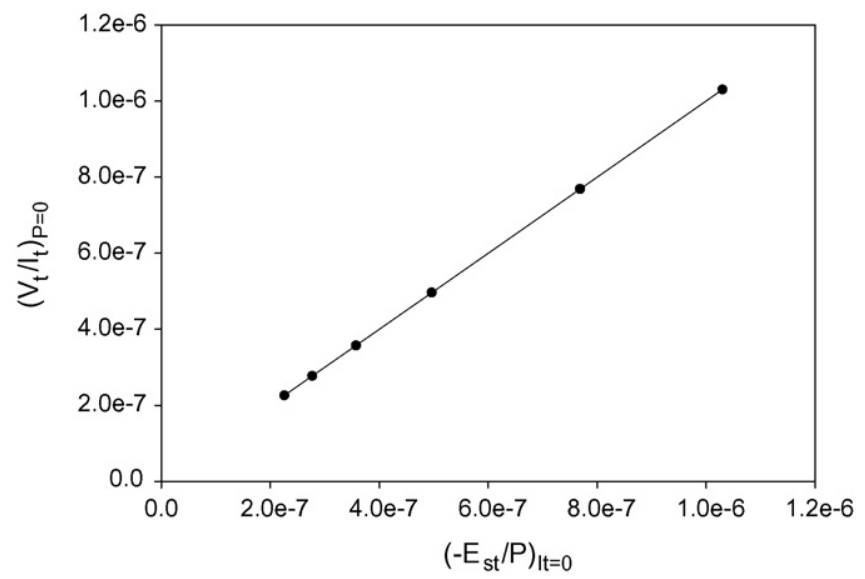

Fig. 10. Variation of $\left(V_{\mathrm{t}} / I_{\mathrm{t}}\right)_{P=0}$ as a function of $\left(\left|E_{\mathrm{st}}\right| / P\right)_{I_{\mathrm{t}}=0}$ at $D_{\mathrm{h}}=10^{-6} \mathrm{~m}$, $d=D_{\mathrm{h}} / 10$, and $I=10^{-3} \mathrm{M}$. Discrete symbols, from right to left, represent the values for $H / W=1,1 / 4,1 / 8,1 / 12,1 / 16,1 / 20$.

reveals that if $D_{\mathrm{e}} \rightarrow \infty$ (or infinitely thin double layer), $R_{\mathrm{vis}} \rightarrow 1$, that is, the electroviscous effect is absent. On the other hand, as $D_{\mathrm{e}} \rightarrow 0, R_{\text {vis }}$ approaches a constant value, which depends upon the value of $D_{\mathrm{e}}$. Some references reported that $R_{\mathrm{vis}}$ has a local maximum as $D_{\mathrm{e}}$ varies $[26,27,42]$. This was not observed in

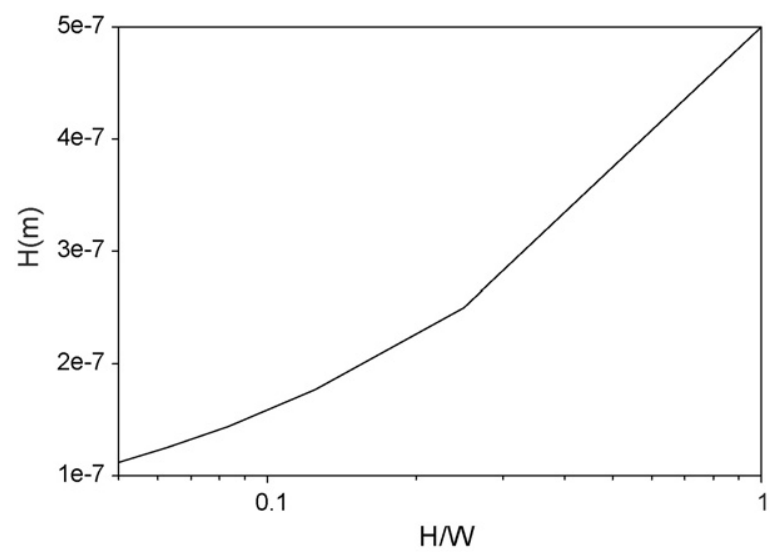

Fig. 11. Variation of semi-minor axis $H$ as a function of aspect ratio $(H / W)$ for the case when the cross-sectional area is fixed at $7.85 \times 10^{-13} \mathrm{~m}^{2}$. this and in previous studies [37]. Burgreen and Nakache [26] pointed out that if the zeta potential is sufficiently high, the local maximum is absent. As can be seen in Table 1, all the absolute values of surface potential are higher than $25 \mathrm{mV}$ in our examples, and increases with the decrease in $D_{\mathrm{e}}$, and, therefore, the local maximum does not exist.

As pointed out by Sørensen and Koefood [28], regardless of the geometry of a microchannel and the distribution of fixed charge in the membrane layer, the Onsager relation

$$
\left(\frac{V_{\mathrm{t}}}{I_{\mathrm{t}}}\right)_{P=0}=-\left(\frac{E_{\mathrm{st}}}{P}\right)_{I_{\mathrm{t}}=0}
$$

needs to be satisfied. This is justified in Fig. 10.
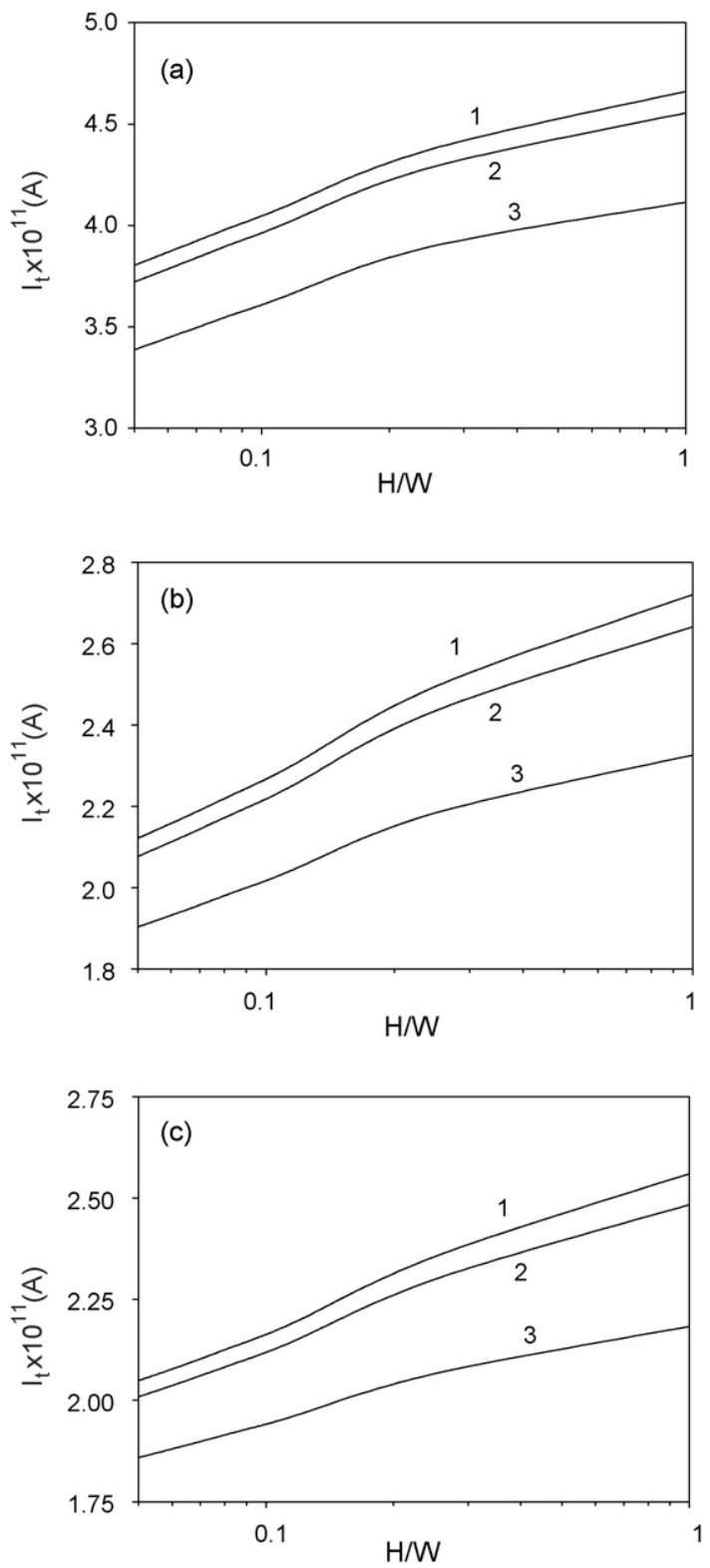

Fig. 12. Variation of total current $I_{\mathrm{t}}$ as a function of aspect ratio $(H / W)$ at various membrane thickness $d_{\mathrm{c}}$ and ionic strength $I$ for the case when cross-sectional area is fixed, $E_{z}=1000 \mathrm{~V} / \mathrm{m}, P=0 \mathrm{~N} / \mathrm{m}^{3}$, and $D_{\mathrm{hc}}=10^{-6} \mathrm{~m}$. (a) $I=10^{-3} \mathrm{M}$, (b) $I=10^{-4} \mathrm{M}$ and (c) $I=10^{-5} \mathrm{M}$. Curve $1, d_{\mathrm{c}}=D_{\mathrm{hc}} / 50 ; 2, d_{\mathrm{c}}=D_{\mathrm{hc}} / 40 ; 3$, $d_{\mathrm{c}}=D_{\mathrm{hc}} / 20$. 

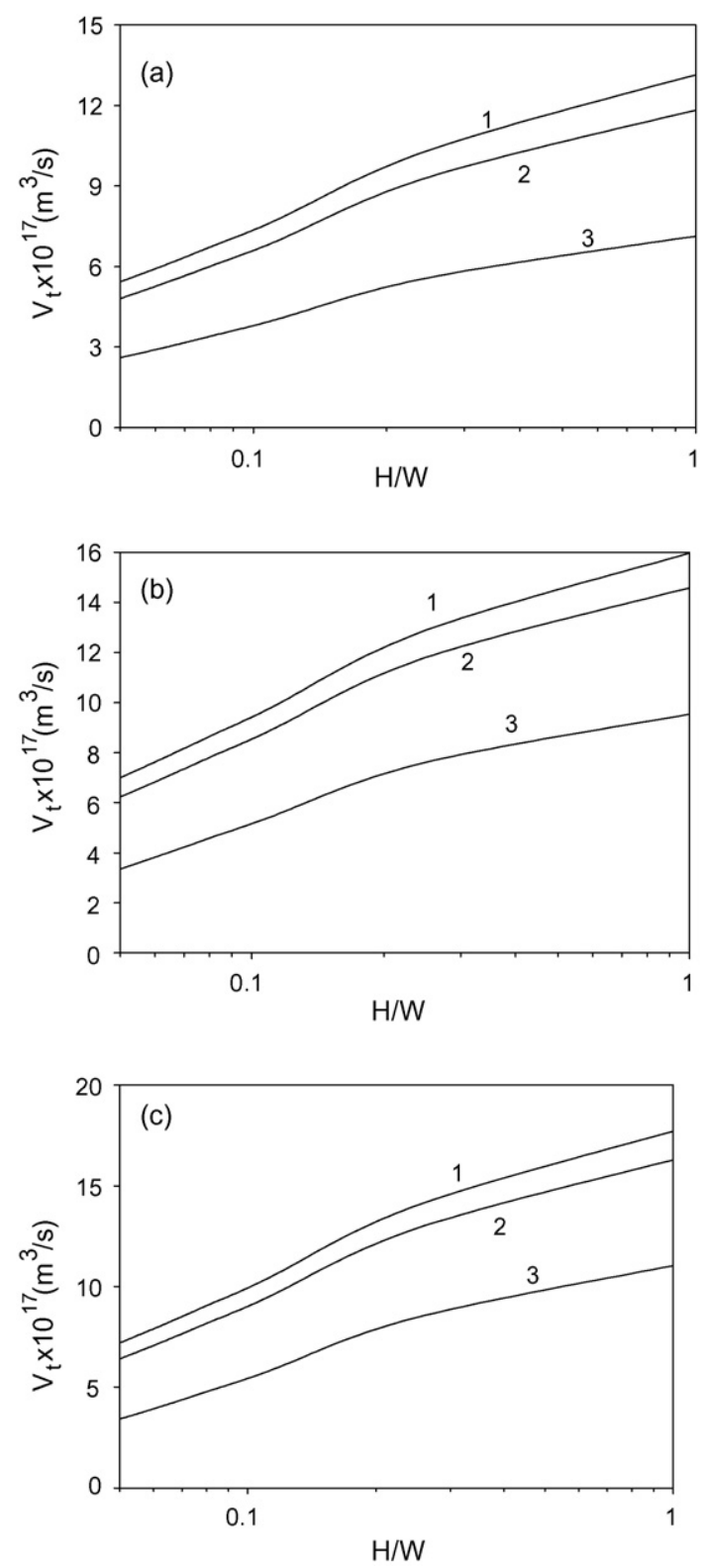

Fig. 13. Variation of total volumetric flow rate $V_{\mathrm{t}}$ as a function of aspect ratio $(H / W)$ at various $d_{\mathrm{c}}$ and $I$ for the case of Fig. 12.

In subsequent discussions the cross-sectional area of a microchannel is fixed at $7.85 \times 10^{-13} \mathrm{~m}^{2}$. Let $D_{\mathrm{hc}}\left(=10^{-6} \mathrm{~m}\right)$ and $d_{\mathrm{c}}$ be, respectively, the diameter of the corresponding cylindrical microchannel (i.e., $H / W=1$ ) and the scaled thickness of the membrane layer. The influences of $(H / W), d_{\mathrm{c}}$, and ionic strength $I$ on the electrokinetic behavior of a microchannel are examined. As illustrated in Fig. 11, if the cross-sectional area of microchannel is fixed, the smaller the $(H / W)$ the narrower it is, that is, the smaller the $H$. Fig. 12 shows the variation of the total current $I_{\mathrm{t}}$ as a function of the aspect ratio $(H / W)$ at various membrane thickness $d_{\mathrm{c}}$ and ionic strength $I$ for the case when the cross-sectional area is fixed, and the corresponding variation in the total volumetric flow rate $V_{\mathrm{t}}$ is illustrated in Fig. 13. Fig. 12 indicates that, for the same values of $(H / W)$ and $d_{\mathrm{c}}, I_{\mathrm{t}}$ increases with the increase in $I$, and for the same value of $I, I_{\mathrm{t}}$ increases with the increase in $d_{\mathrm{c}}$. These behaviors can be explained by the same reasoning as that employed in the discussion of Fig. 5. However, the qualitative behavior of $I_{\mathrm{t}}$ at various levels of $I$ is different from that observed in Fig. 5. Fig. 12 reveals that for the range of the ionic strength considered, if the value of $I$ is fixed, $I_{\mathrm{t}}$ declines with the decrease in $(H / W)$. This is because if the cross-sectional area of a microchannel is fixed, the smaller the $(H / W)$ the narrower is a microchannel, and the more significant the viscous effect. According to Fig. 13, for a given value of $(H / W)$, if the value of $d_{\mathrm{c}}$ is fixed, $I_{\mathrm{t}}$ increases with the decrease in $I$, and if the value of $I$ is fixed, the $V_{\mathrm{t}}$ increases with the decrease
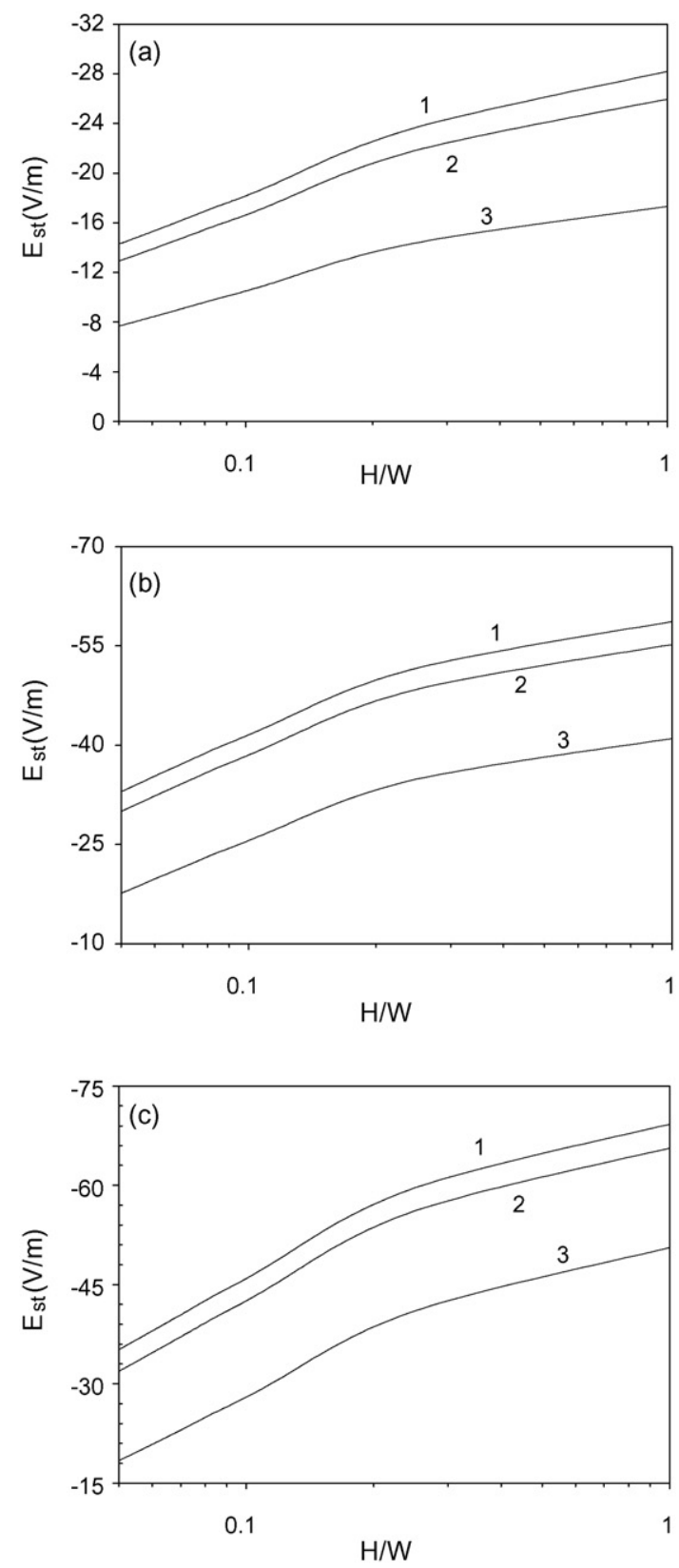

Fig. 14. Variation of streaming potential $E_{\mathrm{st}}$ as a function of aspect ratio $(H / W)$ at various membrane thickness $d_{\mathrm{c}}$ and ionic strength $I$ for the case when cross-sectional area is fixed and $P=10^{7} \mathrm{~N} / \mathrm{m}^{3}, D_{\mathrm{hc}}=10^{-6} \mathrm{~m}$. (a) $I=10^{-3} \mathrm{M}$, (b) $I=10^{-4} \mathrm{M}$ and (c) $I=10^{-5} \mathrm{M}$. Curve $1, d_{\mathrm{c}}=D_{\mathrm{hc}} / 50 ; 2, d_{\mathrm{c}}=D_{\mathrm{hc}} / 40 ; 3$, $d_{\mathrm{c}}=D_{\mathrm{hc}} / 20$. 

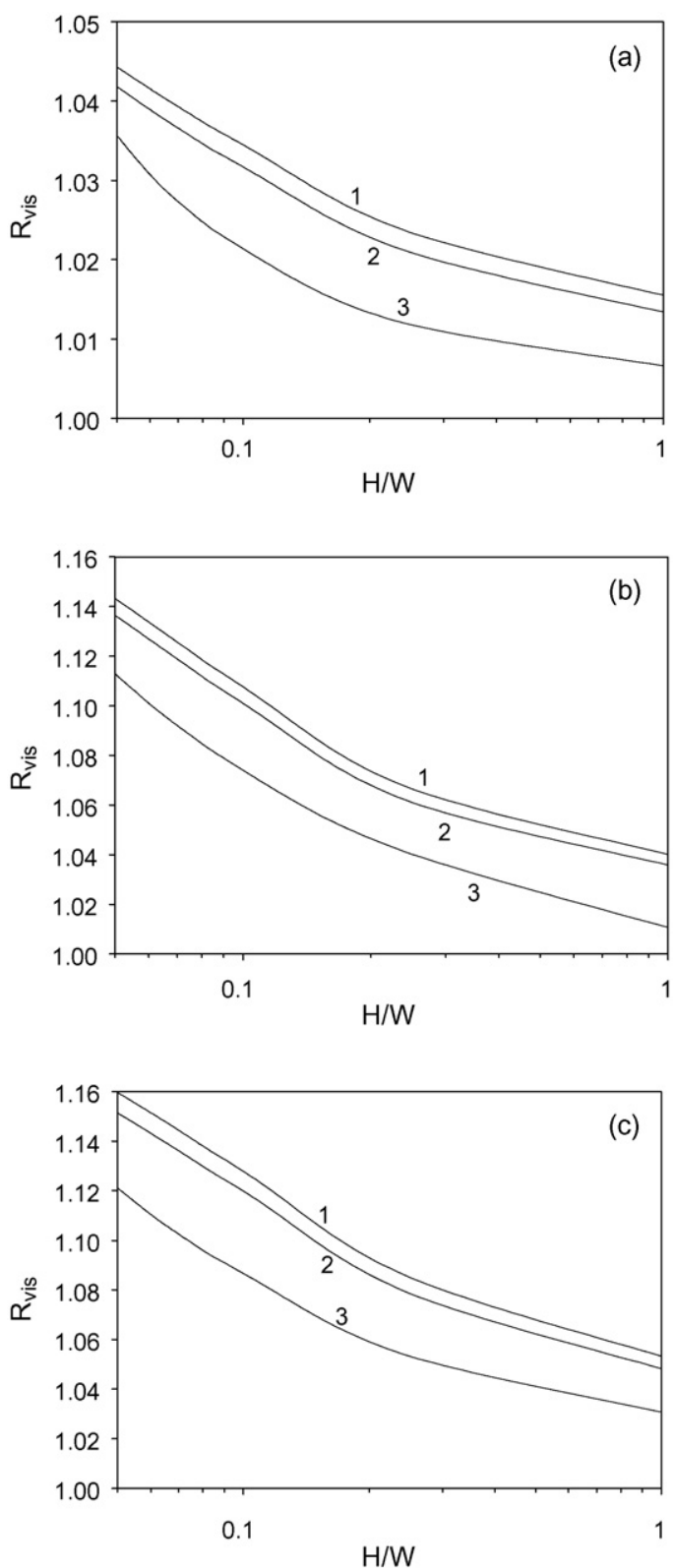

Fig. 15. Variation of electroviscous parameter $R_{\mathrm{vis}}$ as a function of aspect ratio $(H / W)$ at various membrane thickness $d_{\mathrm{c}}$ and ionic strength $I$ for the case of Fig. 14.

in $d_{\mathrm{c}}$. These behaviors can be explained by the same reasoning as that employed in the discussion of Fig. 6, except for all the three levels of $I$ considered, because viscous effect dominates, $V_{\mathrm{t}}$ increases with the increase in $(H / W)$.

The variations of the streaming potential $E_{\mathrm{st}}$ as a function of the aspect ratio $(H / W)$ at various combinations of $d_{\mathrm{c}}$ and $I$ for the case when the cross-sectional area of a microchannel is fixed are illustrated in Fig. 14, and the corresponding variations in $R_{\text {vis }}$ are presented in Fig. 15. When a pressure gradient is applied and the value of $(H / W)$ is fixed, both the decrease in $I$ and that in $d_{\mathrm{c}}$ lead to an increase in $\left|E_{\mathrm{st}}\right|$. This is because the smaller the value of $I$ the wider is the distribution of counterions, which implies that they are easier to be driven by an applied pressure gradient. This leads
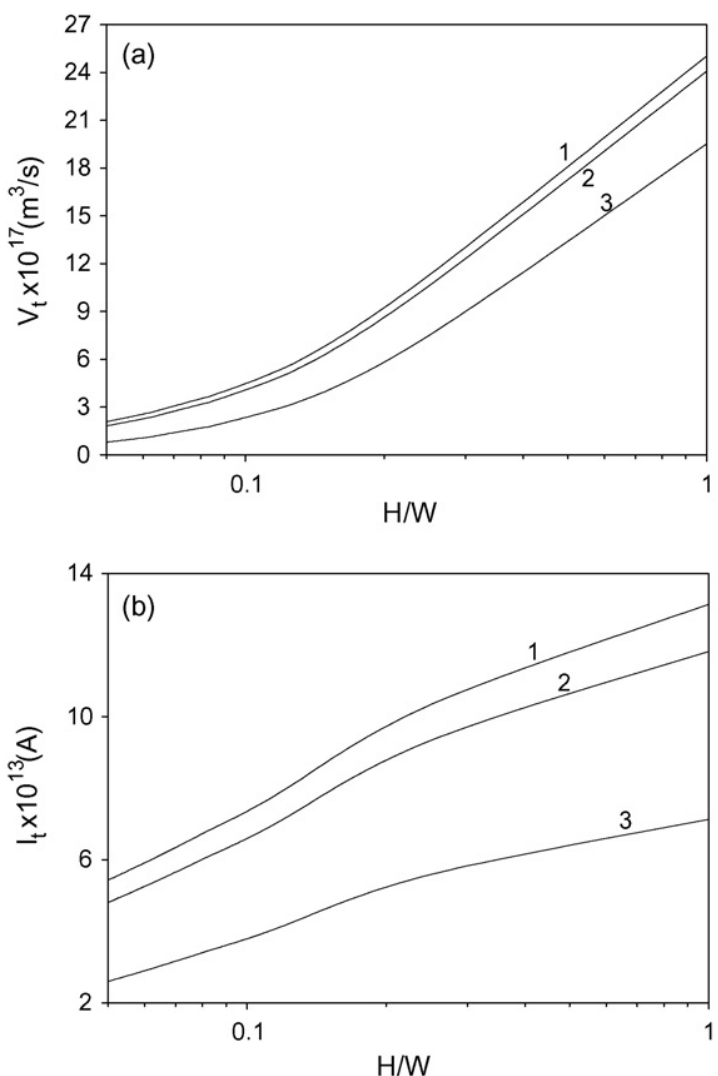

Fig. 16. Variation of total volumetric flow rate $V_{\mathrm{t}}$ (a) and total current $I_{\mathrm{t}}$ (b) as a function of aspect ratio $(H / W)$ at various membrane thickness $d_{\mathrm{c}}$ for the case when cross-sectional area is fixed, $E_{z}=0 \mathrm{~V} / \mathrm{m}, P=10^{7} \mathrm{~N} / \mathrm{m}^{3}, D_{\mathrm{hc}}=10^{-6} \mathrm{~m}$, and $I=10^{-3} \mathrm{M}$. Curve $1, d_{\mathrm{c}}=D_{\mathrm{hc}} / 50 ; 2, d_{\mathrm{c}}=D_{\mathrm{hc}} / 40 ; 3, d_{\mathrm{c}}=D_{\mathrm{hc}} / 20$.

to a larger $I_{\mathrm{t}}$, and therefore, a larger $\left|E_{\mathrm{st}}\right|$ is necessary to establish an equilibrium, as is seen in Fig. 14. Since the induced electric field yields a liquid flow which is in the opposite direction as that arising from the applied pressure gradient, the larger the $\left|E_{\mathrm{st}}\right|$, the smaller the value of $V_{\mathrm{t}}$ at which $I_{\mathrm{t}}=0$, which implies a larger $R_{\text {vis }}$, as is seen in Fig. 15. If the membrane layer is thin, the corresponding hydrodynamic retardation is small, the ions are easier to be driven by an applied pressure gradient, and therefore, both $\left|E_{\mathrm{st}}\right|$ and $R_{\mathrm{vis}}$ become larger. Figs. 14 and 15 indicate that for the levels of ionic strength considered, $\left|E_{\mathrm{st}}\right|$ increases with the increase in $(H / W)$, but the reverse is true for $R_{\mathrm{vis}}$. For the case when only a pressure gradient is applied, an increase in $(H / W)$ yields a wider microchannel, $V_{\mathrm{t}}$ is larger, so is $I_{\mathrm{t}}$, as is seen in Fig. 16(a) and (b), so that a larger $E_{\text {st }}$ is necessary to have it balanced. Note that the behavior of the variation of $V_{\mathrm{t}}$ at $I_{\mathrm{t}}=0$ as $(H / W)$ varies, shown in Fig. 17 , is the same as that when $E_{\text {st }}$ is absent, as illustrated in Fig. 16. This implies that the induced electric field is not strong enough to influence the behavior of $V_{\mathrm{t}}$ as $(H / W)$ varies, and, therefore, at equilibrium, the smaller the $(H / W)$ the narrower a microchannel, the more significant its viscous effect, and $V_{\mathrm{t}}$ is smaller, which implies that $R_{\mathrm{vis}}$ is larger. On the other hand, the strength of the induced electric field when the value of $D_{\mathrm{h}}$ is fixed is stronger enough. Since the behavior of $V_{\mathrm{t}}$ as $(H / W)$ varies is influenced by $\left|E_{\mathrm{st}}\right|$, the larger the $\left|E_{\mathrm{st}}\right|$ the smaller the $R_{\text {vis. }}$. 


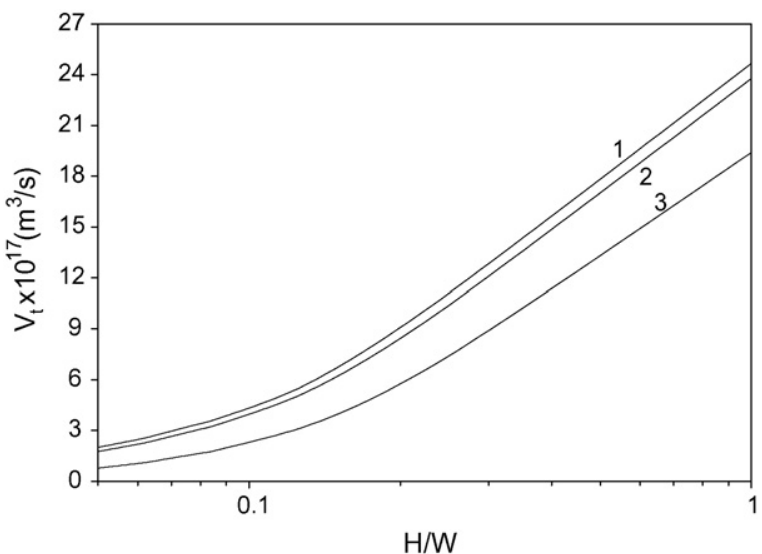

Fig. 17. Variation of total volumetric flow rate $V_{\mathrm{t}}$ as a function of aspect ratio $(H / W)$ at various membrane thickness $d_{\mathrm{c}}$ for the case when cross-sectional area is fixed, $E_{\mathrm{z}}=E_{\mathrm{st}}, P=10^{7} \mathrm{~N} / \mathrm{m}^{3}, D_{\mathrm{hc}}=10^{-6} \mathrm{~m}$, and $I=10^{-3} \mathrm{M}$. Curve 1 , $d_{\mathrm{c}}=D_{\mathrm{hc}} / 50 ; 2, d_{\mathrm{c}}=D_{\mathrm{hc}} / 40 ; 3, d_{\mathrm{c}}=D_{\mathrm{hc}} / 20$

\section{Acknowledgments}

This work is supported by the Department of Economics of the Republic of China under grant 93-EC-17-A-09-S1-019 and the National Science Council of the Republic of China.

\section{References}

[1] I. Kobayashi, S. Mukataka, M. Nakajima, Ind. Eng. Chem. Res. 44 (2005) 5852.

[2] I. Kobayashi, S. Mukataka, M. Nakajima, Langmuir 21 (2005) 5722.

[3] Q.Y. Xu, M. Najajima, B.P. Binks, Colloids Surf. A 262 (2005) 94.

[4] T. Kawakatsu, Y. Kikuchi, M. Nakajima, J. Am. Oil. Chem. Soc. 74 (1997) 317.

[5] S. Sugiura, M. Nakajima, S. Iwamoto, M. Seki, Langmuir 17 (2001) 5562.

[6] T. Nakashima, M. Shimizu, M. Kukizaki, Key Eng. Mater. 61 (1991) 113.

[7] S. Sugiura, M. Nakajima, J. Tong, H. Nabetani, M. Seki, J. Colloid Interf. Sci. 227 (2000) 95.

[8] I. Kobayashi, M. Nakajima, K. Chun, Y. Kikuchi, H. Fujita, Am. Inst. Chem. Eng. J. 48 (8) (2002) 1639.

[9] S.L. Anna, N. Bontoux, H.A. Stone, Appl. Phys. Lett. 82 (3) (2003) 364.

[10] D.R. Link, S.L. Anna, D.A. Weitz, H.A. Stone, Phys. Rev. Lett. 92 (2004) 054503-054511.
[11] M. Kumemura, T. Korenaga, Anal. Chim. Acta 558 (2006) 75

[12] J.P. Brody, P. Yager, Sens. Actuators A 58 (1997) 13.

[13] A.E. Kamholz, B.H. Weigl, B.A. Finlayson, P. Yager, Anal. Chem. 71 (1999) 5340.

[14] M. Tokeshi, T. Minagawa, T. Kitamori, Anal. Chem. 72 (2000) 1711.

[15] V.S. Nagineni, S. Zhao, A. Potluri, Y. Liang, U. Siriwardane, N.V. Seetala, J. Fang, J. Palmer, D. Kuila, Ind. Eng. Chem. Res. 44 (2005) 5602.

[16] R. Srinivasan, I.M. Hsing, P.E. Berger, K.F. Jensen, S.L. Firebaugh, M.A. Schmidt, M.P. Harold, J.J. Lerou, J.F. Ryley, Am. Inst. Chem. Eng. J. 43 (1997) 3059.

[17] W. Ehrfeld, V. Hessel, H. Lowe, Microreactors: New Technology for Modern Chemistry, Wiley-VCH, Weinheim, Germany, 2000.

[18] J. Ju, C. Zeng, L. Zhang, N. Xu, Chem. Eng. J. 116 (2006) 115.

[19] X.N. Zhang, S. Stefanick, F.J. Villani, Org. Process Res. Dev. 8 (2004) 455.

[20] J.L. Cohen, D.J. Volpe, D.A. Westly, A. Pechenik, H.D. Abruna, Langmuir 21 (2005) 3544.

[21] H.L. Maynard, J.P. Meyers, J. Vac. Sci. Technol. B 20 (2002) 1287.

[22] E.R. Choban, L.J. Markoski, A. Wieckowski, P.J.A. Kenis, J. Power Sources 128 (2004) 54.

[23] R. Ferrigno, A.D. Stroock, T.D. Clark, M. Mayer, G.M. Whitesides, J. Am. Chem. Soc. 124 (2002) 12930.

[24] J.L. Cohen, D.A. Westly, A. Pechenick, H.D. Abrunã, J. Power Sources 139 (2005) 96.

[25] S.K. Ryi, J.S. Park, S.H. Choi, S.H. Cho, S.H. Kim, Chem. Eng. J. 113 (2005) 47.

[26] D. Burgreen, F.R. Nakache, J. Phys. Chem. 68 (1964) 1084.

[27] C.L. Rice, R. Whitehead, J. Phys. Chem. 69 (1965) 4017.

[28] T.S. Sørensen, J. Koefoed, J. Chem. Soc. Faraday Trans. 270 (1974) 665.

[29] R.J. Yang, L.M. Fu, Y.C. Lin, J. Colloid Interf. Sci. 239 (2001) 98.

[30] J.P. Hsu, C.Y. Kao, S.J. Tseng, C.J. Chen, J. Colloid Interf. Sci. 248 (2002) 176.

[31] J. Yang, D.Y. Kwok, Langmuir 19 (2003) 1047.

[32] H. Ohshima, S. Ohki, Biophys. J. 47 (1985) 673.

[33] J.H. Keh, Y.C. Liu, J. Colloid Interf. Sci. 172 (1995) 222.

[34] E. Donath, A. Voigt, J. Colloid Interf. Sci. 109 (1986) 122

[35] H. Ohshima, T. Kondo, J. Colloid Interf. Sci. 135 (1990) 443.

[36] S.J. Tseng, C.Y. Kao, J.P. Hsu, Electrophoresis 21 (2000) 3541.

[37] J.P. Hsu, C.Y. Kao, J. Phys. Chem. B 105 (2001) 8135.

[38] J.H. Masliyah, G. Neale, K. Malysa, T.G.M. Van De Ven, Chem. Eng. Sci. 42 (1987) 245.

[39] FlexPDE, Version 2.22, PED Solutions Inc., USA.

[40] J.D. Ferry, Viscoelastic Properties of Polymers, 3rd ed., Wiley, New York, 1980.

[41] G.M. Mala, D. Li, C. Werner, H.J. Jacobasch, Y.B. Ning, Int. J. Heat Fluid Flow 18 (1997) 489.

[42] C. Yang, D. Li, J. Colloid Interf. Sci. 194 (1997) 95. 